\title{
Enhanced Desorption Ionization Using Oxidizing Electrosprays
}

\author{
Marcela Nefliu and R. Graham Cooks \\ Department of Chemistry, Purdue University, West Lafayette, Indiana, USA \\ Colin Moore \\ Chemtura Corporation, Middlebury, Connecticut, USA
}

\begin{abstract}
A signal enhancement of two orders of magnitude was achieved when reactive desorption electrospray ionization (DESI) was used to investigate copper(II) dibutyl dithiocarbamate, $\mathrm{Cu}(\mathrm{II})\left(\mathrm{bu}_{2} \mathrm{dtc}\right)_{2}$, found in a specialized polymer. $\mathrm{Cu}$ (II) was oxidized to $\mathrm{Cu}$ (III) during the DESI experiment by oxidants in the spray solvent. Such oxidants could be present or formed during electrospray (e.g., $\mathrm{O}_{2}$ ) or deliberately added to the spray solvent (this approach is called reactive DESI). When a strong oxidizing agent (e.g., iodine) was added to the spray solvent, the signal increased by two orders of magnitude relative to the pure solvent spray. The correlation between the standard reduction potential of the oxidant and the signal intensity and signal to noise ratio of the product ion for various reagents, was tested and discussed. The observed DESI enhancements in rates of oxidation are not observed in homogeneous solution. The major peaks in the collision induced dissociation (CID) spectrum of the complex ion $\left[\mathrm{Cu}(\mathrm{III})\left(\mathrm{bu}_{2} \mathrm{dtc}\right)_{2}\right]^{+}$were identified using isotopic distributions and $\mathrm{MS}^{3}$ data. (J Am Soc Mass Spectrom 2006, 17, 1091-1095) (c) 2006 American Society for Mass Spectrometry
\end{abstract}

$\mathrm{D}$ esorption electrospray ionization (DESI) [1,2] is a soft ionization technique which uses electrosprayed solvent to desorb ionized condensed phase analytes under ambient conditions. The method can be used for the rapid, sensitive, and specific trace analysis of analytes in a variety of complex materials without sample preparation. Application areas already demonstrated include pharmaceutical preparations [3-5], natural products [6], drug metabolites [7], intact tissue analysis [2], explosives [8], thin-layer chromatography [9], proteolyzed solutions [2], and synthetic polymers [10]. DESI is a heterogeneous process involving charged solution-phase droplets interacting with a condensed phase sample to yield gas-phase ions. It is therefore straightforward to add solution-phase reagents to the spray solvent to mediate the interfacial chemistry. This capability, sometimes called reactive DESI, has been used to perform selective ligand/protein binding [1], to stereoselectively generate covalent cyclic boronates from 1,2-diols [11] and to perform Eberlin transacetalization of acylium ions (Eberlin, M. N., unpublished results). These instances emphasize the analogy between DESI and chemical ionization (CI), even though the former employs solution-phase rather

Published online June 2, 2006

Address reprint requests to Dr. R. G. Cooks, Department of Chemistry, Purdue University, 560 Oval Drive, West Lafayette, IN 47907-2084. E-mail: cooks@purdue.edu than gas-phase reagents as is done in the conventional CI experiment.

We demonstrate in this paper that redox reactions can be used to advantage in DESI analysis (as they have previously been so used in electrospray ionization [12, 13]). We report a very large enhancement in the efficiency of ionization of a particular metal complex present in a specialized layered ethylene/propylene/ diene polymer [14] by using redox spray reagents to assist in ionization. The analyte investigated is the neutral copper(II) dibutyl dithiocarbamate complex, $\mathrm{Cu}(\mathrm{II})\left(\mathrm{bu}_{2} \mathrm{dtc}\right)_{2}$, which is ionized to $\left[\mathrm{Cu}(\mathrm{III})\left(\mathrm{bu}_{2} \mathrm{dtc}\right)_{2}\right]^{+}$ $(\mathrm{m} / \mathrm{z} 471)$ during the DESI experiment (eq 1). Electrochemical processes accompanying electrospray ionization (ESI) were first recognized by Kebarle et al. [12] and thoroughly investigated by Van Berkel and co-

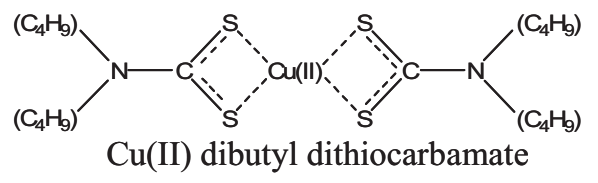

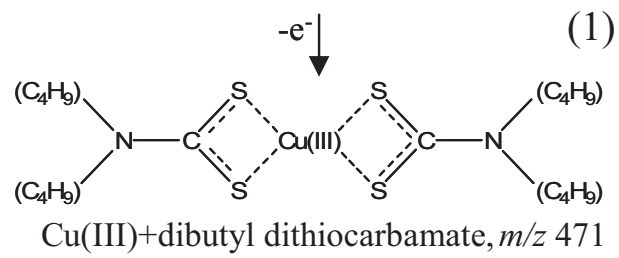


workers $[13,15]$. These processes can lead to the ionization, through one-electron processes, of certain neutral molecules present in the electrosprayed sample. Both reduction $[16,17]$ and oxidation reactions $[18,19]$ have been exploited in the case of organometallic complexes and aromatic compounds. Ionization efficiency can be increased by chemically or electrochemically generating preformed ions before infusion into the ESI, either by adding external redox agents [20] or by passing the solution through an on-line or off-line electrochemical cell [16].

In the DESI experiment the analyte does not come into physical contact with the anode (i.e., the syringe needle to which the voltage is applied). Rather, it is chemically oxidized by species naturally formed or present in the electrospray (e.g., $\mathrm{O}_{2}$ from ambient air and from the oxidation of water [17]) or by reagents deliberately added to the spray solvent. Having a chemical reaction as the basis for the ionization process, the problem of enhancing the ionization efficiency is a problem in chemical reactivity. By deliberately adding a reagent of choice to the spray solvent as an alternative to using physical source parameters (e.g., electrospray voltage to increase the oxygen production [17]) to control the ionization process, we combine straightforward solution-phase redox chemistry with the ease with which any reagent can be delivered in DESI to the surface of the ambient sample.

We report here enhancement of the ionization efficiency of copper dibutyl dithiocarbamate, $\mathrm{Cu}(\mathrm{II})\left(\mathrm{bu}_{2} \mathrm{dtc}\right)_{2}$, by two orders of magnitude by adding oxidizing agents to the spray solvent. From the isotopic distribution of fragment ions and $\mathrm{MS}^{3}$ data, we also attempt to identify all the major peaks in the collision induced dissociation (CID) spectrum of the complex ion $\left[\mathrm{Cu}(\mathrm{III})\left(\mathrm{bu}_{2} \mathrm{dtc}\right)_{2}\right]^{+}$. Note that the copper complex is examined as an additive in a bulk polymer.

\section{Experimental}

The experiments were carried out using a commercial Thermo Finnigan LTQ (San Jose, CA) linear ion trap mass spectrometer. The DESI source was home-built, as previously described [2] but is similar to the commercial source of Prosolia Inc. (Indianapolis, IN).

The acetonitrile $(\mathrm{MeCN})$ was from Mallinckrodt Baker Inc. (Phillipsburg, NJ) and used as received. All the other reagents were from Sigma-Aldrich Inc. (Milwaukee, WI) except for 1,2-naphthoquinone (Distillation Products Industries, Eastman Chemical Company, Rochester, NY) and iodine (Mallinckrodt Baker Inc.). The polymer, a layered ethylene propylene diene monomer (EPDM) rubber, was supplied by Chemtura Corporation (Middlebury, CT) and used without any chemical processing. The $\mathrm{Cu}$ (II) dibutyl dithiocarbamate is an aging product resulting from the reaction of tetrabutyl thiuram disulfide and copper from copper 2-mercaptobenzothiazolate [14], which are additives. The experiments were performed on newly exposed

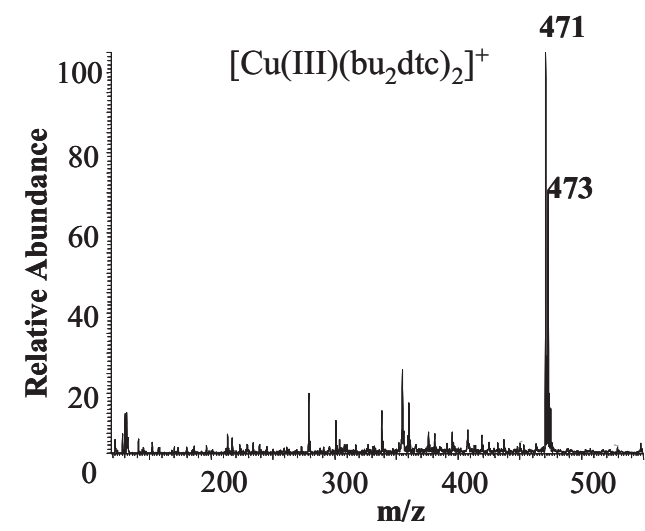

Figure 1. Positive DESI mass spectrum of the polymer sample sprayed with $\mathrm{I}_{2}\left(2 \times 10^{-4} \mathrm{M}\right)$ in MeCN.

polymer surface obtained by slicing the upper rubber layer into thin pieces. Small pieces of about $4 \mathrm{~mm}^{2}$ were then used for each measurement.

Each sample was placed $5 \mathrm{~mm}$ away from the MS inlet capillary and then electrosprayed from a distance of $2.5 \mathrm{~mm}$, at a flow rate of $5 \mu \mathrm{Lmin}^{-1}$ and an incidence angle of $60^{\circ}$. The syringe needle was held at $5 \mathrm{kV}$, the inlet capillary at $15 \mathrm{~V}$, and the tube lens at $65 \mathrm{~V}$. The nebulizing gas pressure (nitrogen) measured at the gas tank, was 200 psi.

\section{Results and Discussion}

The ionization of $\mathrm{Cu}$ (II) dibutyl dithiocarbamate in the ESI source involves simple oxidation of $\mathrm{Cu}$ (II) to $\mathrm{Cu}$ (III) $[14,21]$. Such behavior is not unusual for transitionmetal dithiocarbamates of the type $\mathrm{M}\left(\mathrm{R}_{2} \mathrm{dtc}\right)_{2}$ for which the high oxidation state complexes $\left[\mathrm{M}\left(\mathrm{R}_{2} \mathrm{dtc}\right)_{2}\right]^{+}$are remarkably stable [20]. In the DESI source we expect a similar ionization mechanism for the copper complex. Indeed, upon sample examination using $\mathrm{MeCN}$ as the spray solvent, a small signal corresponding to $\left[\mathrm{Cu}(\mathrm{III})\left(\mathrm{bu}_{2} \mathrm{dtc}\right)_{2}\right]^{+}$was generated. The chemical species most likely responsible for this redox process is oxygen absorbed from the atmosphere, and/or generated at the syringe needle by water electrolysis. Signal variation with the electrospray (ES) voltage, known to favor the electrochemical reactions inside the spray solvent, was negligible in the tested range of 0 to $7 \mathrm{kV}$, suggesting only minor contributions of this process to the total oxygen content. Changing the nature of the oxidizing species by adding a reagent of choice to the spray solvent is therefore the best method to enhance the ionization efficiency and, as our results demonstrate, this is also simple and very effective. This technique, a type of reactive DESI experiment, is demonstrated for iodine (Figure 1), which produces a signal increase of two orders of magnitude. A possible correlation between the signal intensity and the oxidizing strength of the reagent was investigated using eight organic and inorganic oxidants. Table 1 shows the intensities of the copper complex ion, the signal to noise ratios $(\mathrm{S} / \mathrm{N})$ and 
Table 1. Signal abundances, signal to noise ratios and half-wave and standard reduction potentials for various spray reagents

\begin{tabular}{|c|c|c|c|c|c|}
\hline Spray reagent & $\begin{array}{c}\text { DESI signal } \\
\text { intensity }(\mathrm{RSD} \%)^{\mathrm{a}}\end{array}$ & DESI S/N (RSD\%) ${ }^{a}$ & $\begin{array}{c}\text { ESSI signal } \\
\text { intensity }(\mathrm{RSD} \%)^{\mathrm{a}}\end{array}$ & ESSI S/N (RSD\%) ${ }^{a}$ & $E(V)$ \\
\hline Acetonitrile (oxygen) & $170(100)$ & $7.1(110)$ & $2.7(47)$ & $2.8(34)$ & $-0.82^{b}[23]$ \\
\hline 1-Chloroanthraquinone ${ }^{c}$ & $810(120)$ & $5.5(21)$ & $0.068(40)$ & $2.2(33)$ & $-0.90^{d}[24]$ \\
\hline 1,2-Naphthoquinone ${ }^{c}$ & $17(50)$ & $30(70)$ & $8.1(3.9)$ & $7.8(3.5)$ & $-0.56^{\mathrm{e}}[25]$ \\
\hline 1,4-Benzoquinone ${ }^{c}$ & $460(1.8)$ & $27(3.6)$ & $8.5(55)$ & $2.7(40)$ & $-0.51^{\mathrm{e}}[25]$ \\
\hline $\begin{array}{l}\text { Tetrafluoro-1,4- } \\
\text { benzoquinone }^{f}\end{array}$ & $1400(92)$ & $69(0.12)$ & $120(8.3)$ & $110(6.4)$ & $-0.04^{\mathrm{e}}[25]$ \\
\hline $\begin{array}{l}\text { Tetrachloro-1,4- } \\
\text { benzoquinone }^{f}\end{array}$ & $1200(74)$ & $90(19)$ & $170(13)$ & $140(32)$ & $0.01^{\mathrm{e}}[25]$ \\
\hline lodine $^{f}$ & $98,000(130)$ & $170(24)$ & $54(16)$ & $4.6(21)$ & $0.52^{\mathrm{g}}[26]$ \\
\hline Potassium bichromate $^{f}$ & $130,000(25)$ & $240(1.5)$ & $470(4.6)$ & $30(6.0)$ & $1.36^{\mathrm{h}}[22]$ \\
\hline Hydrogen peroxide ${ }^{c}$ & $400(79)$ & $29(66)$ & $8.2(15)$ & $7.8(11)$ & $1.76^{\mathrm{h}}[22]$ \\
\hline
\end{tabular}

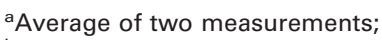

${ }^{b} \mathrm{E}_{1 / 2}$ determined by cyclic voltammetry in MeCN;

c $2.2 \times 10^{-3} \mathrm{M}$ in $\mathrm{MeCN}$;

${ }^{\mathrm{d}} \mathrm{E}_{1 / 2}$ determined by polarography in dimethylformamide;

${ }^{e} \mathrm{E}_{1 / 2}$ determined by polarography in $\mathrm{MeCN}$;

f $2.2 \times 10^{-5} \mathrm{M}$ in $\mathrm{MeCN}$;

${ }^{\mathrm{g}} \mathrm{E}^{0}$ in $\mathrm{MeCN}$;

${ }^{\mathrm{h}} \mathrm{E}^{\mathrm{O}}$ in water.

the half-wave reduction potentials $\left(\mathrm{E}_{1 / 2}\right)$ or standard reduction potentials $\left(E^{0}\right)$ for each reagent. Each spectrum was recorded using a $50 \mathrm{~ms}$ ion injection time into the ion trap, and the signal was averaged over 20 scans. The $\mathrm{S} / \mathrm{N}$ was calculated as the ratio between the intensity of the signal at $\mathrm{m} / \mathrm{z} 471$ and the averaged intensities of five background peaks in the vicinity of the target signal: $m / z 430,440,450,480$, and 500 .

The $E_{1 / 2}$ and $E^{0}$ values were taken from literature and used in this work after a few simplifying assumptions were made. Thus, the half-wave potentials [22] were assumed to equal the standard potentials and the traces of water in the solvent (initially present or collected from atmosphere in the course of the experiment) are presumed to have a negligible effect on the values of the potentials. For potassium bichromate and hydrogen peroxide no studies in non-aqueous media have been found in the surveyed literature and so, $\mathrm{E}^{0}$ in aqueous phase were used. For this reason these two systems should not be directly compared to the rest of the oxidants. Even though we advise the reader to treat these data with caution, we believe that neither the purpose of this work nor the nature of the experiments for which the solvent composition would be difficult to control strictly due to changes in $\mathrm{pH}$ and oxygen content in the ES, require at this point a more accurate set of reference data. All the tested reagents were soluble in unpurified $\mathrm{MeCN}$ at the specified concentrations.

Our results reveal no clear correlation between the oxidative strength of the reagents and the signal abundances. However, a plot of reduction potential versus $\mathrm{S} / \mathrm{N}$ is approximately linear $\left(\mathrm{R}^{2}=0.95\right)$ when hydrogen peroxide and potassium bichromate are excluded from the dataset. The first point of the series was attributed to the pure solvent spray, assuming that oxygen is the active species. A large decrease in the $\mathrm{E}^{0}$ of hydrogen peroxide in $\mathrm{MeCN}$ (as it is also the case for oxygen) could account for the pronounced deviation from the trend established by the other reagents.

The rather poor reproducibility of the data reflected in the relative standard deviation (RSD) values could be due to small variations in sample flatness and position relative to the spray plume and MS inlet, to the small number of replicates, and to a possible nonhomogeneous distribution of the copper(II) complex inside the polymer layer. $\mathrm{S} / \mathrm{N}$ values were thus compared along with absolute signals, as they better reflect the signal enhancement. The difference in the reagent concentration for the most effective oxidants (see Table 1) relative to the less effective ones had a negligible effect on the relative signal intensities.

It is possible to map the spatial distribution of molecules by DESI [2]. The analyte distribution was therefore investigated by scanning the sample surface with three different solvent sprays containing tet-

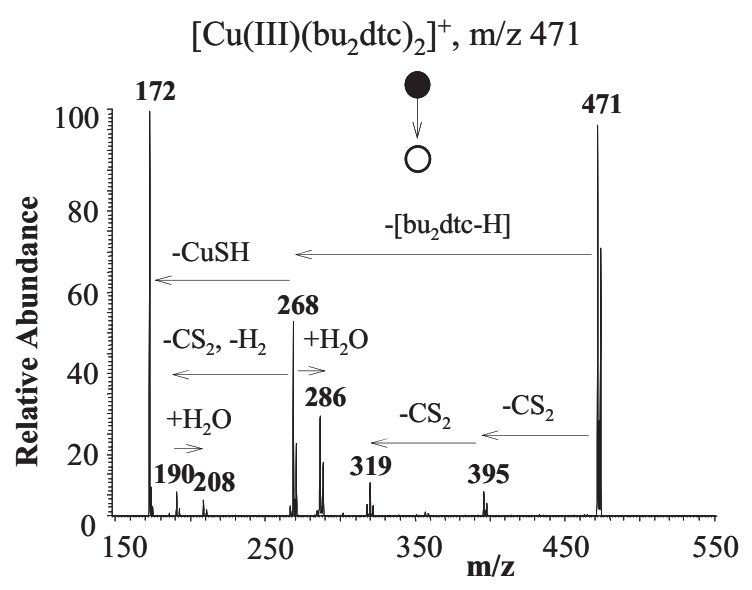

Figure 2. Product ion $\mathrm{MS}^{2}$ spectrum of $\left[\mathrm{Cu}(\mathrm{IIII})\left(\mathrm{bu}_{2} \mathrm{dtc}\right)_{2}\right]^{+}$. 


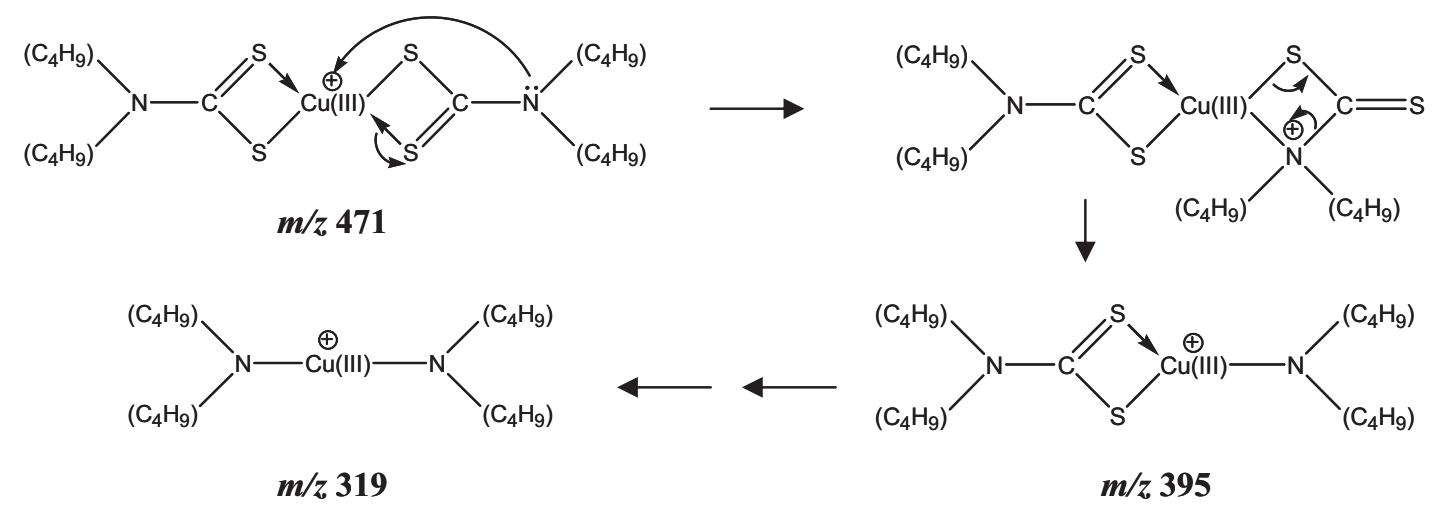

Scheme 1. Fragmentation of $\mathrm{CU}(\mathrm{III})\left(\mathrm{bu}_{2} \mathrm{dtc}\right)_{2}$ cation

rafluoro-1,4-benzoquinone, tetrachloro-1,4-benzoquinone, and iodine. Each time, the sample was examined by manually moving the sample stage in front of the MS inlet with a rate of about $0.1 \mathrm{~mm} \mathrm{~s}^{-1}$. Six data points were collected over a distance of about $7 \mathrm{~mm}$ so that each value averaged the signal collected over a distance of about $1 \mathrm{~mm}$, which is the diameter of the sampling surface area of the spray plume. The RSD values calculated for each scanning experiment using the six data points were between 23 and $46 \%$ for the absolute signals and between 10 and $26 \%$ for the $\mathrm{S} / \mathrm{N}$ suggesting that point-to-point variability could make some contribution to the overall precision of the experiment.

To verify if the selected reagents have a similar behavior in the homogeneous solution phase to that seen in the heterogeneous DESI experiment, we allowed them to react in equal concentrations and for equal duration (2 min), with an extract of the copper(II) dithiocarbamate in acetonitrile solution. The mixture was then analyzed by electrosonic spray ionizationmass spectrometry (ESSI-MS). The data were measured and analyzed in a manner similar to that described above. Most of the reagents had little effect on the signal intensity as shown in Table 1 and no clear relationship was found between the $\mathrm{S} / \mathrm{N}$ values and the reduction potentials. Interestingly, though, other peaks containing copper were observed in many of the spectra, suggesting reactivity of the reagent towards the copper complex. For example, the dimer of the copper complex, $\left[\mathrm{Cu}\left(\mathrm{bu}_{2} \mathrm{dtc}\right)_{2}\right]_{2}{ }^{+}(\mathrm{m} / \mathrm{z}$ 942) was identified in the potassium bichromate and tetrafluoro-1,4-benzoquinone spectra. In the DESI experiments such products were much less abundant than the target ion and so, they passed unnoticed. It thus appears that the oxidants do not act only as innocent one-electron acceptors and that a more complex chemistry accounts for the uncorrelated solution phase data. Similar reactivity towards transition-metal dithiocarbamates has been previously demonstrated for nitrosonium tetrafluoroborate [20]. The simple one-electron-transfer is the favored process and in the much smaller time window available during the DESI experiments (in the order of milliseconds) compared to the ESSI experiments $(2 \mathrm{~min})$, the $\left[\mathrm{Cu}(\mathrm{III})\left(\mathrm{bu}_{2} \mathrm{dtc}\right)_{2}\right]^{+}$predominates. A possible catalytic effect of the surface on the formation of the $\left[\mathrm{Cu}(\mathrm{III})\left(\mathrm{bu}_{2} \mathrm{dtc}\right)_{2}\right]^{+}$could also explain the differences in the product yield observed between the two experiments. More dilute solutions or shorter reaction times could be necessary to match DESI results. This is therefore, a case where the heterogeneous phase experiments offer access to a more simple chemistry than the homogeneous solution phase experiments. We believe that for the system studied here, the standard reduction potentials could still be used for optimum reagent selection in DESI experiments, and the signal increase is sufficiently large to disregard the competitive reactions.

Metal complexes with dithiocarbamates have been previously investigated by ESI-MS and the observed fragments identified $[14,21]$. We attempted to elucidate the fragmentation pattern of the complex $\left[\mathrm{Cu}(\mathrm{III})\left(\mathrm{bu}_{2} \mathrm{dtc}\right)_{2}\right]^{+}$to confirm the reported results and to identify new fragments. Thus, the major peaks in the $\mathrm{MS}^{2}$ spectrum of the $\mathrm{Cu}(\mathrm{III})$ complex ion were identified from both the isotopic distributions and from the corresponding $\mathrm{MS}^{3}$ spectra.

The fragmentation pattern is presented in the product ion $\mathrm{MS}^{2}$ spectrum shown in Figure 2 and the calculated, as well as measured, isotopic distributions for each ion are listed in Table 1S (Supplemental Material section, which can be found in the electronic version of this article). Dissociation of the precursor ion was achieved by collisions with helium gas in the ion trap, at collision energies of 22 to $22.5 \%$ (expressed in terms of the manufacturer's nominal relative collision energy). The isolation window was set at \pm 3 Thomson centered upon $\mathrm{m} / \mathrm{z} 472$, to cover the entire isotopic distribution. Our results confirm that the previously identified [14] peaks at $m / z 172,190,268$, and 286, have the assignments presented in Table 1S. The signal at $\mathrm{m} / \mathrm{z} 286$ is the in-trap hydration product of the ion at $\mathrm{m} / \mathrm{z} 268$ and we believe that likewise, the signal at $m / z 208$ is due to in-trap hydration of the ion at $\mathrm{m} / \mathrm{z} 190$. These water adducts are much more abundant in the ion trap mass spectrometer than they are in the Q-TOF instrument 
used to acquire the spectra reported in reference [14], due to the long path length and low-energy of the trapped, oscillating ions [27]. Similar increased ion hydration relative to that observed using other mass spectrometers has been noted previously for other metal complexes, including metal carbonyls [27, 28]. In reference 14, exact mass measurement was used to identify the fragments in the $\mathrm{MS}^{2}$ spectrum however, for the $\mathrm{m} / \mathrm{z} 395$ fragment ion, this measurement suggested an anomalous molecular formula, i.e., $\mathrm{C}_{20} \mathrm{H}_{31} \mathrm{~N}_{2} \mathrm{~S}_{3}$. We suggest that this fragment ion is in fact $\mathrm{C}_{17} \mathrm{H}_{36} \mathrm{~N}_{2} \mathrm{~S}_{2} \mathrm{Cu}$ and resulted from loss of carbon disulfide $\left(\mathrm{CS}_{2}\right)$ from the intact copper complex. Our conclusion is based on the similarity between the calculated and the measured isotopic distribution. The result is also consistent with the exact mass data [14] since the theoretical mass for $\mathrm{C}_{17} \mathrm{H}_{36} \mathrm{~N}_{2} \mathrm{~S}_{2} \mathrm{Cu}$ is 395.1616 and the measured mass was 395.1639 Da. These data show the value of having both $\mathrm{MS}^{\mathrm{n}}$ and exact mass measurement information available for elucidating structural problems.

A mechanism for the $\mathrm{CS}_{2}$ loss is proposed in Scheme 1. The lowest $\mathrm{m} / \mathrm{z}$ that could be measured in our CID spectrum was $\mathrm{m} / \mathrm{z} 150$ and so, lower mass fragments could not be observed.

\section{Conclusions}

This study shows that an increase in the desorption ionization efficiency of two orders of magnitude was possible for the $\mathrm{Cu}$ (II) dibutyl dithiocarbamate, when a reactive spray containing an oxidizing agent was used. Parallel measurements made in homogeneous solution showed negligible oxidation for most of the reagents (as well as some minor products not seen in the DESI experiment) suggesting that the rate of oxidation is increased by interfacial processes, which are being further explored. This demonstration of the ability to use chemical reagents to alter the selectivity of DESI, suggests the possibility of using solution-phase chemistry without sample pretreatment, to enhance sensitivity. The dissociation pathways were also identified.

\section{Acknowledgments}

This work was supported by the Office of Naval Research under grant number BAA ONR 04-024. Research support from Prosolia Inc. and Chemtura Corporation is also gratefully acknowledged.

\section{References}

1. Takats, Z.; Wiseman, J. M.; Gologan, B.; Cooks, R. G. Mass Spectrometry Sampling under Ambient Conditions with Desorption Electrospray Ionization. Science 2004, 306, 471-473.

2. Takats, Z.; Wiseman, J. M.; Cooks, R. G. Ambient Mass Spectrometry Using Desorption Electrospray Ionization (DESI): Instrumentation, Mechanisms, and Applications in Forensics, Chemistry, and Biology. J. Mass Spectrom. 2005, 40, 1261-1275.
3. Chen, H.; Talaty, N. N.; Takats, Z.; Cooks, R. G. Desorption Electrospray Ionization Mass Spectrometry for High-Throughput Analysis of Pharmaceutical Samples in the Ambient Environment. Anal. Chem. 2005, 77, 6915-6927.

4. Williams, J. P.; Scrivens, J. H. Rapid Accurate Mass Desorption Electrospray Ionization Tandem Mass Spectrometry of Pharmaceutical Samples. Rapid Commun. Mass Spectrom. 2005, 19, 3643-3650.

5. Kauppila, T. J.; Wiseman, J. M.; Ketola, R. A.; Kotiaho, T.; Cooks, R. G.; Kostiainen, R. Desorption Electrospray Ionization Mass Spectrometry for the Analysis of Pharmaceuticals and Metabolites. Rapid Commun. Mass Spectrom. 2006, 20, 387-392.

6. Talaty, N. N.; Chen, H.; Takats, Z.; Cooks, R. G. Desorption Electrospray Ionization (DESI) of Natural Products for Alkaloid Detection in Plant Tissue and High Throughput Analysis of Pharmaceutical Samples. Analyst 2005, 130, 1624-1633.

7. Chen, H.; Pan, Z; Talaty, N. N.; Cooks, R. G.; Raftery, D. Combining Desorption Electrospray Ionization Mass Spectrometry and Nuclear Magnetic Resonance for Differential Metabolomics without Sample Separation. Rapid Commun. Mass Spectrom. 2005, in press.

8. Cotte-Rodriguez, I.; Takats, Z: Talaty, N. N.; Chen, H.; Cooks, R. G. Desorption Electrospray Ionization of Explosives on Surfaces: Sensitivity and Selectivity Enhancement by Reactive Desorption Electrospray Ionization. Anal. Chem. 2005, 77, 6755-6764.

9. Van Berkel, G. J.; Ford, M. J.; Deibel, M. A. Thin-Layer Chromatography and Mass Spectrometry Coupled Using Desorption Electrospray Ionization. Anal. Chem. 2005, 77, 1207-1215.

10. Nefliu, M.; Venter, M.; Cooks, R. G. Desorption Electrospray Ionization and Electrosonic Spray Ionization for Solid- and Solution-Phase Analysis of Industrial Polymers. Chem. Commun. 2006, 8, 888-890.

11. Chen, H.; Cotte-Rodriguez, I.; Cooks, R. G. Cis-Diol Functional Group Recognition by Reactive Desorption Electrospray Ionization (DESI). Chem. Commun. 2006, 6, 597-599.

12. Blades, A. T.; Ikonomou, M. G.; Kebarle, P. Mechanism of Electrospray Mass Spectrometry. Electrospray as an Electrolysis Cell. Anal. Chem. 1991, 63, 2109-2114.

13. Van Berkel, G. J.; McLuckey, S. A.; Glish, G. L. Electrochemical Origin of Radical Cations Observed in Electrospray Ionization Mass Spectra. Anal. Chem. 1992, 64, 1586-1593.

14. Moore, C.; McKeown, P. LCMS/MS and TOF-SIMS Identification of the Color Bodies on the Surface of a Polymer. J. Am. Soc. Mass Spectrom. 2005, 16, 295-301.

15. de la Mora, J. F.; Van Berkel, G. J.; Enke, C. G.; Cole, R. B.; MartinezSanchez, M.; Fenn, J. B. Electrochemical Processes in Electrospray Mass Spectrometry. I. Mass Spectrom. 2000, 35, 939-952.

16. Gun, I: Modestov, A. Rinaldo, P. Ovadia, L. Reduction of $\left[\left(\mathrm{C}_{5} \mathrm{Me}_{5}\right)_{2} \mathrm{Mo}_{2} \mathrm{O}_{5}\right]$ and $\left[\left(\mathrm{C}_{5} \mathrm{Me}_{5}\right)_{2} \mathrm{Mo}_{2} \mathrm{O}_{4}\right]$ in Methanol/Water Trifluoroacetate Solutions Investigated by Combined On-Line ElectrochemistryIonization Mass Spectrometry. Eur. I. Inorg. Chem. 2003, 12, 2264-2272.

17. Van Berkel, G. J. Electrolytic Deposition of Metals on to the HighVoltage Contact in an Electrospray Emitter: Implications for Gas-Phase Ion Formation. J. Mass Spectrom. 2000, 35, 773-783.

18. Van Berkel, G. J.; McLuckey, S. A.; Glish, G. L. Electrospray Ionization of Porphyrins Using a Quadrupole Ion Trap for Mass Analysis. Anal. Chem. 1991, 63, 1098-1109.

19. Xu, X.; Nolan, S. P.; Cole, R. B. Electrochemical Oxidation and Nucleophilic Addition Reactions of Metallocenes in Electrospray Mass Spectrometry. Anal. Chem. 1994, 66, 119-125.

20. Bond, A. M.; Colton, R.; D'Agostino, A.; Harvey, J.; Traeger, J. C. Electrospray Mass Spectrometric Study of the Nature and Liability of Cationic Complexes Generated by the Reaction of Solutions of Neutral Iron(III), Cobalt(III), Nickel(II), and Copper(II) Dithiocarbamates with Nitrosonium Tetrafluoroborate. Inorg. Chem. 1993, 32, 3952-3956.

21. Schoener, D. F.; Olsen, M. A.; Cummings, P. G.; Basic C. Electrospray Ionization of Neutral Metal Dithiocarbamate Complexes Using InSource Oxidation. J. Mass Spectrom. 1999, 34, 1069-1078.

22. Bard, A. J. Electrochemical Methods: Fundamentals and Applications; John Wiley and Sons Inc.: New York, 2001; pp 178-180.

23. Peover, M. E.; White, B. S. Electrolytic Reduction of Oxygen in Aprotic Solvents: The Superoxide Ion. Electrochim. Acta 1996, 11, 1061-1067.

24. Brodsky, A. E.; Gordienko, L. L.; Degtiarev, L. S. Cathodic Reduction of Some Aromatic Compounds to Free Anion-Radicals. Electrochim. Acta. 1968, 13, 1095-1100.

25. Peover, M. E. A Polarographic Investigation into the Redox Behavior of Quinones: The Roles of Electron Affinity and Solvent. J. Chem. Soc. 1962, 4540-4549.

26. Milazzo, G.; Caroli, S. Tables of Standard Electrode Potentials; Wiley and Sons: New York, 1978.

27. Louris, J. N.; Cooks, R. G.; Syka, J. E. P.; Kelley, P. E.; Stafford, J. G. C.; Todd, J. F. J. Instrumentation, Applications, and Energy Deposition in Quadrupole Ion-Trap Tandem Mass Spectrometry. Anal. Chem. 1987, 59, 1677-1685.

28. Cooks, R. G.; Glish, G. L.; Kaiser, J. R. E.; McLuckey, S. A. Ion Trap Mass Spectrometry. Chem. Eng. News 1991, 69, 26-41. 